\title{
Perspectivas de docentes y gestores educativos ante las medidas adoptadas en Paraguay debido al covid-19 1
}

\section{Teachers' and educational administrators' views on the measures adopted in Paraguay due to covid-19}

DOI: $10.46932 / \mathrm{sfjdv} 2 \mathrm{n} 2-080$

Received in: March 1st, 2021

Accepted in: May 30th, 2021

\section{Valentina Canese}

Ph.D. en Currículum e Instrucción - Arizona State University - Master en Educación con énfasis en Lengua y Políticas Lingüísticas - San Diego State University - Licenciada en Lengua Inglesa Universidad Nacional de Asunción. Directora - Instituto Superior de Lenguas, Universidad Nacional de Asunción.

\section{Juan Ignacio Mereles}

MSc. en Elaboración, Gestión y Evaluación de Proyectos de Investigación Científica - Universidad Nacional de Asunción. Licenciado en Ciencias Mención Matemática Estadística- Universidad Nacional de Asunción. Coordinador de Postgrado e Investigación - Universidad Nacional de Asunción. E-mail: jimereles@facen.una.py.

\section{Jessica Amarilla}

Magister en Educación - University of Western Australia - Licenciatura en Lengua Inglesa Universidad Nacional de Asunción - Docente - Universidad Nacional de Asunción

E-mail: isl-jamarilla@ fil.una.py

\section{RESUMEN}

Debido a la pandemia causada por el COVID-19, gobiernos en todo el mundo han tomado medidas que han afectado a los sistemas educativos, interrumpiendo las clases presenciales de al menos $70 \%$ de las instituciones, para evitar la propagación del virus (UNESCO 2020). En Paraguay se dio un cierre abrupto y temprano. Este estudio presenta un análisis de las perspectivas institucionales de gestores y docentes con respecto a los desafíos presentados teniendo en cuenta la preparación que existía en relación a factores tales como infraestructura y acceso a la tecnología, capacitación docente, limitaciones de estudiantes y padres. Se utilizó una metodología mixta cuan-cual con cuestionarios de preguntas cerradas y abiertas. La muestra incluyó a 1030 docentes y 110 gestores educativos de instituciones públicas y privadas de todos los niveles de todo el país. El estudio muestra que a inicios de las medidas adoptadas por el gobierno las instituciones educativas tuvieron inconvenientes en el desarrollo de las clases a distancia. Las principales dificultades fueron el limitado acceso a internet, la escasa capacitación en el uso de las TIC educativas y el acceso a ellas. De igual manera, minimizar la deserción estudiantil y conservar la calidad de la educación representan los mayores desafíos para la continuidad del proceso educativo. Estos aspectos demuestran la necesidad de un mayor y mejor acceso a las tecnologías educativas por parte de los principales actores educativos del país.

Palabras Clave: Instituciones Educativas - COVID-19-Gestores - Docentes - Desafíos

\footnotetext{
${ }^{1}$ Derivado del proyecto de investigación: Experiencias de actores educativos ante la pandemia del Covid-19: financiación propia.
} 


\begin{abstract}
Due to the COVID-19 pandemic, governments around the world have taken measures that have affected education systems, disrupting face-to-face classes in at least $70 \%$ of institutions, to prevent the spread of the virus (UNESCO 2020). In Paraguay there was an abrupt and early closure. This study presents an analysis of teachers' and educational administrators' institutional perspectives with respect to the challenges presented, taking into account the preparation that existed considering factors such as infrastructure and access to technology, teacher training, student and parent limitations. A mixed quanqual approach was followed using closed and open-ended questionnaires. The sample included 1030 teachers and 110 educational administrators from public and private institutions at all levels throughout the country. The study shows that at the beginning of the measures adopted by the government, educational institutions had difficulties in the development of distance learning. The main difficulties were limited access to the Internet, poor training in the use of and access to educational ICT. Similarly, minimizing student dropout and preserving the quality of education represent the greatest challenges to the continuity of the educational process. These aspects demonstrate the need for greater and better access to educational technologies by the country's main educational actors.
\end{abstract}

Keywords: Educational Institutions - COVID-19 - Administrators- Teachers - Challenges

\title{
1 INTRODUCCIÓN
}

La pandemia del COVID-19 trajo consigo numerosas disrupciones en el ámbito económico, social y educativo en todo el mundo. Declarada como tal el 13 de marzo por la Organización Mundial de la Salud (OMS), se establecieron medidas sanitarias para controlar la propagación del virus, siendo una de estas el distanciamiento físico (WHO, 2020). Debido a las medidas promulgadas por la OMS, escuelas y universidades cerraron puertas y adoptaron nuevas estrategias para dar continuidad a la educación afectando a alrededor de 1.2 billones de estudiantes en todo el mundo (UNESCO, 2020a). Sin embargo, el impacto de la pandemia en la educación no tiene precedentes y se estiman costos a corto y largo plazo (Grupo Banco Mundial, 2020). El cierre de organizaciones educativas trae consigo efectos negativos como el aumento del abandono escolar y reducción de salarios de estudiantes en el futuro (Sanz, Sainz Gonzalez \& Capilla, 2020), interrupción de la educación y costo económico para las familias entre otros (UNESCO, 2020b). Los efectos negativos se evidencian y acentúan debido a la desigualdad de los sistemas educativos (CEPAL, 2020; Cifuentes-Faura, 2020; Monasterio \& Briceño, 2020).

Paraguay registró su primer caso de coronavirus el 7 de marzo del 2020 y posteriormente se tomaron medidas de aislamiento y confinamiento recomendadas por el Ministerio de Salud Pública y Bienestar Social (MSPBS, 2020). Las medidas de confinamiento llevaron al cierre de las instituciones educativas desde el 10 de marzo y estrategias tecnológicas fueron adoptadas para la continuidad de la educación (Britez, 2020). De esta manera, el Ministerio de Educación y Ciencia (MEC) desarrolló un plan para dar continuidad a la educación considerando los diferentes contextos de los estudiantes paraguayos denominado "Tu escuela en casa" (MEC, 2020). De la misma manera, las instituciones superiores, 
siguiendo indicaciones de la Organización de Estados Iberoamericanos para la Educación, la Ciencia y la Cultura, adaptaron contenidos de clases al sistema virtual (OEI, 2020). Para tal efecto, el Consejo Nacional de Educación Superior (CONES), estableció pautas en cuanto a la aplicación de herramientas digitales en instituciones superiores aprobando alrededor de 2453 programas académicos hasta el 27 de julio del 2020 (CONES, 2020).

Las perspectivas y respuestas de los distintos actores educativos en relación a la pandemia del COVID-19 y cómo estos están afrontando la situación desde sus respectivos contextos es de suma importancia. El cambio abrupto de circunstancias ha llevado a la comunidad educativa a implementar medidas sin precedentes por lo cual no se cuentan con datos sobre cómo se está sobrellevando el proceso educativo. Este estudio se enfoca en analizar las perspectivas institucionales de gestores y docentes con respecto a los desafíos presentados teniendo en cuenta la preparación que existía en relación a factores tales como

infraestructura y acceso a la tecnología, capacitación docente, limitaciones de estudiantes y padres.

\section{MATERIAL Y MÉTODOS}

Se utilizó una metodología mixta cuan-cual con cuestionarios de preguntas cerradas y abiertas adaptados de Wozney, Venkatesh y Abrami (2006) a las condiciones de confinamiento por el COVID-19, seguido por grupos focales con participantes voluntarios. El estudio se realizó durante la cuarta semana de suspensión de actividades académicas (finales de abril e inicios de mayo) y la muestra incluyó a 1030 docentes y 110 gestores de instituciones públicas, privadas y subvencionadas de todos los niveles y de todas las regiones del país a excepción de Alto Paraguay. El 70\% de los docentes y $71 \%$ de los gestores educativos que participaron de la encuesta residen en Asunción y en el Departamento Central, mientras que los restantes residen en los demás departamentos del país. El cuestionario docente consistió de 37 preguntas incluyendo algunas referentes al acceso a la tecnología, capacidades y capacitación docente, tiempo dedicado a la planificación, tipos de actividades utilizadas, medios de comunicación e instrucción, acompañamiento de los padres, posibilidades y dificultades encontradas. Las preguntas abiertas apuntaron a registrar las perspectivas de los docentes no registradas a través de los demás ítems y poder profundizar sobre los desafíos presentados por situación extraordinaria presentada por el COVID-19. Por su parte, el cuestionario para gestores educativos contó con 32 preguntas. Ambos cuestionarios fueron realizados a través de una herramienta para encuestas online y distribuidos por medios digitales como listas de correos, redes sociales tales como Facebook y grupos de Whatsapp.

Los datos fueron analizados utilizando estadística descriptiva univariante (recuentos y porcentajes a través de tablas y gráficos estadísticos) y pruebas de asociación o diferencia de proporciones como la 
Chi cuadrada. Para la manipulación y ordenación de los datos se utilizó la planilla electrónica Excel, mientras que para el análisis estadístico se utilizó el software estadístico R (R Core Team, 2020). Los datos cualitativos fueron analizados utilizando estrategias de análisis cualitativo de contenido a través de la codificación de los datos y la categorización de los mismos en tres grandes categorías: “dificultades”, “medidas adoptadas" y “oportunidades". Dentro de estas se pudo identificar sub-categorías que incluyeron primeramente aspectos referentes a la conectividad, las finanzas, la migración digital, los miembros de la comunidad, la incertidumbre y la calidad. Entre las medidas se encontraron subcategorías referentes a la continuidad de las acciones, adaptación de acuerdo a las posibilidades, continuar con las acciones, priorizar la salud, enfocarse en el currículum, suspender las clases, brindar asistencia económica y materiales impresos a las familias así como acompañamiento al proceso educativo. Finalmente, en relación a las oportunidades, se identificaron subcategorías referentes a la oportunidad de capacitarse, de invertir en infraestructura, de replantear todo el sistema y de realizar la migración digital.

\section{RESULTADOS}

El $65 \%$ de los docentes y el $62 \%$ de los gestores educativos que han participado del estudio se desempeñan en instituciones de enseñanza públicas y el resto en instituciones privadas y subvencionadas. La mayoría de los docentes (49,3\%) enseñan en universidades e institutos superiores, el 25,9\% en el nivel secundario y en menores proporciones en niveles iniciales, primer ciclo y segundo ciclo. Por su parte, los gestores educativos participantes en este estudio mayormente se desempeñan en el nivel escolar básico y nivel medio del sistema educativo nacional. Alrededor del 58\% de los docentes participantes cuentan con títulos de postgrado, 26,6\% cuenta con licenciatura y los demás cuentan con título de profesorado y técnico superior.

Una amplia mayoría de docentes (99\%) ha indicado contar con al menos un teléfono inteligente en el hogar; esta es la principal herramienta con la que el docente mantiene comunicación con los demás miembros educativos. Por otro lado, las respuestas de los docentes evidenciaron una menor tenencia de computadoras que de teléfonos celulares. Un alto porcentaje manifestó contar con solo una computadora para toda la familia $(48 \%)$ o incluso no contar con alguna $(7,4 \%)$, potenciando de esta manera la dificultad de realizar tareas relacionadas a la preparación de materiales didácticos, asentamiento de planillas electrónicas por medios digitales, entre otras. No obstante, un porcentaje no bajo de docentes $(35,2 \%)$ ha manifestado contar con al menos al menos una computadora para cada uno de los miembros del hogar en edad escolar para la realización de las diferentes actividades académicas. Estos docentes se localizan en mayor porcentaje en la capital, Asunción y en el Departamentos Central. 
Se encontró un alto porcentaje $(62,4 \%)$ de docentes que manifestaron contar con conexión a internet a través de los servicios de fibra óptica, cable, antena o wifi, que normalmente tienen costos elevados en comparación a los paquetes de datos móviles. Siguiendo la misma línea, una importante proporción de docentes $(37,7 \%)$ indicó que la única conexión a internet con que cuentan es a través del teléfono móvil. Alrededor del 60\% de los participantes docentes expresó que las conexiones a internet con que cuentan en el hogar son ilimitadas, esto apoyado también en los resultados de que la mayoría de los docentes cuentan con servicios de internet por medio de fibra óptica, antena, cable o wifi, que habitualmente son ilimitados. Sin embargo, se destacó que, en la mayoría de los departamentos, a excepción de Central y de Asunción, existen brechas importantes en cuanto a la conectividad a internet $\left(\chi^{2}=81.8, \mathrm{p}<0.001\right)$. En la capital del país y en el departamento Central se evidenciaron altos porcentajes de docentes con conexiones ilimitadas a internet. Totalmente lo contrario se mostró para los demás departamentos, coincidiendo de esta manera con los gestores que respondieron que sus docentes presentan dificultades con el acceso limitado a internet, principalmente en el interior del país.

La mayoría de los docentes que habían tenido alguna capacitación en el uso de las TIC para la enseñanza antes de que se declarase la emergencia sanitaria y el cierre parcial de las instituciones educativas del país respondió estar recibiendo capacitación en el manejo de las TIC. Más del 47\% de los docentes expresó contar con una capacitación de al menos 3 horas antes de la cuarentena. Por otro lado, alrededor del $31 \%$ de aquellos docentes que no recibieron ninguna capacitación antes de la cuarentena, la han recibido una vez declarada la misma. No obstante, se evidenció una alta proporción de docentes que aún no recibieron alguna capacitación en el uso de las TIC al momento de este estudio. Las proporciones difieren de manera significativa $\left(\chi^{2}=211.9, \mathrm{p}<0.001\right)$. En la misma línea, el $65 \%$ de los gestores educativos respondió que sus docentes no habían recibido alguna capacitación en el uso de herramientas tecnológicas antes de que iniciara la cuarentena. Sin embargo, con la cuarentena ya declarada, el 50\% de los directivos indicó que los docentes que se desempeñan en sus respectivas instituciones educativas han recibido capacitación especial sobre las clases virtuales y las distintas herramientas de educación a distancia disponibles. En este sentido, 45 de las 70 instituciones que manifestaron no haber tenido capacitaciones en el uso de las TIC antes de la cuarentena seguían sin hacerlo en las primeras semanas de la cuarentena.

En cuanto a las etapas de relacionamiento del proceso de integración de las TIC en la enseñanza, siguiendo a Wozney, Venkatesh y Abrami (2006), se pudo apreciar que el 13,7\% de los docentes se declararon en la etapa de Conciencia. En esta etapa se considera que el docente es consciente de que la tecnología existe, pero no la ha utilizado y tal vez incluso la está evitando. La posibilidad de usar computadoras produce en el docente ansiedad. Este aspecto coincide con lo manifestado por algunos 
gestores educativos. Sin embargo, en el transcurso de la cuarentena este porcentaje disminuyó significativamente (aproximadamente en un $10 \%, \chi^{2}=54.4, \mathrm{p}<0.001$ ). Se evidenciaron también aumentos leves en las demás etapas de relacionamiento del proceso de integración de las TIC denominadas de Familiaridad, de Adaptación y de Aplicación creativa.

Tabla 1. Canales de comunicación más utilizados por docentes y gestores educativos (\%)

\begin{tabular}{|l|l|l|l|l|l|}
\hline Canales de & \multicolumn{4}{|l}{} & \multicolumn{2}{l|}{ Gestores Educativos } \\
\cline { 2 - 6 } comunicación utilizados & Institución & Estudiantes & Padres & Docentes & $\begin{array}{l}\text { Padres } \\
\text { Estudiantes }\end{array}$ \\
\hline Mensajes de texto & 4,5 & 3,9 & 3,1 & 15,5 & 15,5 \\
\hline WhatsApp & 82,2 & 76,5 & 50,4 & 95,5 & 90,9 \\
\hline Email Educativa & 45,3 & 34,3 & 11,5 & 53,6 & 50,0 \\
\hline $\begin{array}{l}\text { Plataforma } \\
\text { Online }\end{array}$ & 38,1 & 43,5 & 7,4 & 23,6 & 46,4 \\
\hline Videoconferencia & 27,2 & 26,0 & 3,8 & 54,5 & 45,5 \\
\hline
\end{tabular}

En el estudio se pudo observar que el WhatsApp y el correo electrónico son los canales de comunicación más utilizados por los centros de enseñanza donde se desempeñan los docentes para transmitir avisos sobre temas educativos ( $82 \%$ docentes utilizan el primer canal de comunicación mientras que $45 \%$ utilizan el correo electrónico). Del mismo modo, para la comunicación con los estudiantes y con los padres, los docentes utilizan el servicio de mensajería WhatsApp con mayor frecuencia (76\% y 50\%, respectivamente), coincidiendo con la mayoría de los gestores educativos, quienes en más del $90 \%$ expresaron utilizar este medio como el principal para la comunicación con los padres, estudiantes y docentes (Tabla 1). Al inicio de las clases a distancia, más de la mitad de los docentes (55\%) utilizaban actividades sincrónicas como videoconferencias, chats en vivo, entre otras para el desarrollo de las actividades académicas. Se destaca que la mayoría de los que realizaban estas actividades sincrónicas mantenían el horario de clases utilizado en la modalidad presencial (45\% de los docentes), el 32\% indicó que se establecieron horarios de clases adaptados para la contingencia, mientras que $23 \%$ no utilizaban horarios fijos sino más bien realizaban las actividades sincrónicas de acuerdo a la necesidad y a las circunstancias presentadas a medida que se avanzaba con el proceso educativo, nuevo para muchos actores educativos. La videoconferencia es una de las herramientas que frecuentemente utilizan los gestores para mantener el vínculo con sus docentes.

Se observaron variadas dificultades experimentadas por los docentes en esta nueva modalidad de enseñanza, al menos para la gran mayoría, a partir de las medidas adoptadas por el gobierno para hacer frente al COVID-19. Estas dificultades están asociadas con problemas de conexión a internet (39\%), falta de conocimiento en el uso de herramientas tecnológicas (29\%), la falta de soporte técnico (18\%) y de herramientas tecnológicas (17\%). Respecto a la falta de conocimiento en el uso de herramientas tecnológicas y la conexión a internet, también los gestores respondieron que estas son las dificultades más 
visibles encontradas en los docentes, al igual que en los estudiantes. En menor medida también se mostraron dificultades asociadas a la falta de materiales (10\%). Cabe señalar que el $22 \%$ de los docentes indicaron no tener dificultades en este proceso de enseñanza a distancia mediada por las tecnologías. La mayoría de los docentes (52\%) coincidieron en que la principal dificultad vista en los estudiantes para la realización de las actividades escolares propuestas en esta modalidad se centra en problemas de conectividad a internet.

La falta de conocimiento en el uso de herramientas tecnológicas, la falta de tiempo o interés en las tareas propuestas, el limitado acceso a las TIC, la falta de comprensión de las tareas asignadas, en cierto grado dificultades relacionadas a la falta de apoyo de los padres o tutores y la falta de motivación son también dificultades observadas en los estudiantes según los docentes. En esta ocasión solo el $7 \%$ de los docentes manifestó no estar viendo dificultades en los estudiantes para la realización de los trabajos escolares. Se pudo apreciar un aumento significativo en el tiempo que los docentes destinaban para la preparación de las clases y actividades pedagógicas como así también el destinado para la corrección de los trabajos enviados por los estudiantes. No obstante, entre el $6 \%$ y el $11 \%$ expresaron que estas actividades disminuyeron significativamente. Esto puede deberse a que algunas instituciones de educación superior no habían continuado con el proceso educativo por medios digitales o incluso algunas no habían iniciado el año lectivo al inicio de la cuarentena y no estaban volviendo aún a las actividades académicas, tal como lo indicaron también algunos gestores educativos que formaron parte del estudio.

La zona de residencia del docente no fue un factor discriminante para la presencia de dificultades. Es decir, no se evidenciaron diferencias significativas en la manifestación de dificultades por departamentos de residencia $\left(\chi^{2}=4.77, \mathrm{p}=0.09\right)$. Los años de experiencia de los docentes tampoco influyeron en la manifestación de tener dificultades en la enseñanza a distancia en este tiempo de confinamiento $\left(\chi^{2}=5.22, \mathrm{p}=0.73\right)$. Por otro lado, una relación interesante se dio entre la frecuencia del uso de las TIC en las clases habituales y las dificultades en el proceso académico presentadas en esta pandemia. Se observó que cuando mayor es el uso de las TIC, la probabilidad de que manifieste dificultades es menor. Las diferencias en los porcentajes son estadísticamente significativas $\left(\chi^{2}=42.26\right.$, $\mathrm{p}<0.001)$. Del mismo modo, a medida que el docente manifestó tener un nivel avanzado de competencia en el uso de tecnologías informáticas, las probabilidades que tengan dificultades disminuyen $\left(\chi^{2}=55.12\right.$, $\mathrm{p}<0.001)$. Así también, las dificultades fueron observadas en mayores proporciones en docentes que expresaron contar con conexión a internet de manera limitada. No obstante, se apreció un porcentaje alto $(>70 \%)$ de docentes con dificultades a pesar de contar con acceso ilimitado a internet. 
Tabla 2: Clases virtuales antes y entrada la cuarentena por parte de instituciones educativas públicas, privadas y subvencionadas. Distribución porcentual según oferta de clases virtuales antes de la pandemia

\begin{tabular}{|l|l|l|l|l|}
\hline \multicolumn{2}{|c|}{} & \multicolumn{4}{|l|}{$\begin{array}{l}\text { Impartiendo clases virtuales a partir de las } \\
\text { medidas adoptadas por el gobierno }\end{array}$} \\
\cline { 3 - 5 } & Sí & No & Total \\
\hline \multirow{2}{*}{$\begin{array}{l}\text { Se ofrecían ya clases virtuales } \\
\text { antes de la padema }\end{array}$} & Sí & 96 & 4 & 100 \\
\cline { 2 - 5 } & No & 87 & 13 & 100 \\
\hline
\end{tabular}

Por otro lado, en lo que respecta al desafío de mantener los puestos de trabajo en las instituciones educativas, el 13\% de los gestores manifestó que en la institución donde trabajan actualmente no se mantuvo la totalidad de sus funcionarios semanas después de declararse la cuarentena por el COVID-19, siendo las instituciones privadas las más afectadas por este hecho. En promedio, 11 personas fueron cesadas de sus puestos laborales con la entrada en vigencia de la cuarentena, según los participantes gestores. Algunos gestores han indicado incluso que hasta 35 personas quedaron sin trabajo en sus instituciones educativas. Se pudo observar que en el $22 \%$ de las instituciones que participaron en el estudio, los salarios de los empleados se redujeron o sufrieron alguna modificación temporal.

El 87\% de los participantes gestores mencionó que las instituciones donde ofician ofrecieron clases virtuales a partir de las medidas adoptadas por el gobierno, para dar seguimiento al proceso académico. Las direcciones de las instituciones que indicaron no estar dando clases a través de medios digitales argumentaron diferentes motivos entre los que se destacaron la falta de infraestructura tecnológica, falta de capacitación de muchos de los docentes en el uso de herramientas digitales, suspensión temporal de las clases para iniciar con el proceso de transición a la nueva modalidad de enseñanza, entre otros motivos. En esta misma línea, la mayoría de los gestores expresó estar implementando por primera vez estas clases virtuales mediadas por las tecnologías de la información y comunicación. De aquellos que ya estaban implementando clases virtuales antes de la pandemia, una alta proporción la utilizaba como apoyo a las clases presenciales (alrededor del $75 \%$ ).

Por otro lado, el 60\% de los gestores respondieron que en la institución donde trabajan estuvieron ofreciendo descuentos especiales, o algún otro arreglo económico, a los alumnos y padres para alivianar un poco la difícil situación atravesada en un ambiente de total incertidumbre causada por la pandemia. La mitad de los gestores manifestaron su disconformidad con las medidas de suspender las clases presenciales hasta el mes de diciembre del presente año, siendo las instituciones privadas y subvencionadas los que presentaron mayor oposición a esta decisión tomada por el gobierno nacional.

Según el análisis cualitativo de las respuestas abiertas, se identificaron tres grandes categorías: dificultades, medidas adoptadas y oportunidades. En relación a las dificultades, los participantes volvieron 
a referirse principalmente a cuestiones referentes al acceso a la tecnología reportando dificultades con la “conexión a internet y equipamientos tecnológicos... En la gran mayoría de las familias hay un solo celular y hay varios niños que tienen que utilizar para las tareas" o que existe "baja conectividad de internet... [y] falta de dominio de la tecnología para las reuniones virtuales con los docentes, personal administrativo y de servicios".

Así también, los participantes indicaron a las dificultades financieras como cuestiones centrales a considerar en relación no solamente a la conectividad sino a la continuidad de las instituciones educativas privadas. La incertidumbre generada por la suspensión de actividades, especialmente las económicas, llevó a muchos de los gestores a indicar una preocupación muy importante en relación a la capacidad de continuar brindando los servicios educativos si los padres no tienen la capacidad de pagar por el servicio educativo o "no quieren pagar”. Así, uno de los gestores indicó lo siguiente:

Estamos en una incertidumbre total. No tenemos un parámetro a seguir; estamos entre la espada y la pared. Mensualmente perdemos un 20\% del alumnado. Nos obligan en pocas palabras a pagar salarios, IPS (seguro social) no nos espera porque igual hay que pagar servicios básicos [e] internet en más proporción y todo lo demás... Los padres exigen $50 \%$ de descuento pero sabemos que por más que hagamos eso solo un $20 \%$ pagará por la falta de trabajo... en fin muchas cosas más.

Como puede verse en esta cita, la incertidumbre es otro de los temas que fue destacado por todos los miembros de la comunidad educativa, especialmente los gestores quienes tienen que mediar entre las medidas adoptadas por el gobierno y los demás actores. Con respecto a esto, varios de ellos refirieron que los lineamientos presentados tanto por el MEC como por el CONES en un principio no fueron claros y ellos quedaron en la incertidumbre de cómo llevar a cabo la "migración virtual" a la que también se refirieron como una de las principales dificultades al inicio de la pandemia. Este proceso implica llevar todos los procesos tanto educativos como administrativos a un formato para el cual las instituciones no se encontraban preparadas de varias maneras. Entre ellas se encuentra la infraestructura tecnológica, el contar con las plataformas adecuadas que puedan atender a los estudiantes. Así también, parte de esta migración es la siguiente subcategoría referente a los miembros de la comunidad quienes no se encontraban preparados para esta migración.

Primeramente, tanto docentes como gestores notaron que la falta de capacitación es una las dificultades más importantes indicadas en referencia no solamente al personal de las instituciones sino a los estudiantes y sus padres. Por un lado, los docentes como se pudo ver más arriba en los resultados cuantitativos, admiten sus limitaciones respecto a transferir sus lecciones a una modalidad a distancia. Especialmente se refirieron a la organización del tiempo en esta modalidad ya que "ahora no tienes tiempo ni de descanso... los fines de semana ya no son fines de semana porque creen que trabajando desde casa 
estas siempre descansando". Esto lleva a un "desgaste mental" expresado de esta manera por ambos, docentes y gestores.

En relación a los padres y estudiantes, indicaron que éstos tampoco se encontraban preparados para asumir una nueva modalidad educativa. Por un lado, los "padres no tienen la preparación para acompañar a sus hijos en las tareas" fue una de las dificultades manifestadas. Por otro lado, tampoco los estudiantes, a pesar de ser "nativos digitales", se encontraban preparados para realizar las tareas escolares a distancia según lo indicado por los docentes. Así, los docentes indicaron dificultades para que los estudiantes y padres comprendan las consignas enviadas a través de los diversos medios utilizados; y, muchos de ellos indicaron que la comunicación con los estudiantes representa una de las mayores dificultades manifestando que "el mayor desafío es llegar a todos los estudiantes de la misma manera que lo haría en forma presencial”. Esto último guarda estrecha relación con la preocupación de todos los miembros de la comunidad de no solo llegar a todos sino de "cuidar la calidad" del proceso de enseñanzaaprendizaje en este contexto extraordinario.

Con respecto a las medidas adoptadas por las instituciones de acuerdo a lo expresado por los gestores educativos se encuentran primeramente aquellas orientadas a dar continuidad al proceso educativo. Muchos de ellos mencionaron que la migración digital se realiza "de acuerdo a las posibilidades" de las instituciones y de los miembros de la comunidad ya que como se vio más arriba, muchos no cuentan con las condiciones óptimas para hacerlo. Así también mencionaron la necesidad de "adaptarse" a las circunstancias, enfocarse en el currículum y de "priorizar la salud". Por otro lado, algunos mencionaron la necesidad de que el gobierno pueda dar asistencia económica para poder continuar, así como la posibilidad de preparar materiales impresos para facilitar a aquellos que no tienen una conectividad asegurada. Otra medida sugerida por varios de los participantes fue la de suspender las clases por tres meses para volver después del receso de invierno, ya que en ese momento muchos miembros de la comunidad estimaban que para ese entonces ya se podría volver a la presencialidad. Sin embargo, luego de varios meses la situación aún no está en condiciones de volver a clases presenciales. Finalmente, muchos de los gestores indicaron la necesidad de proveer apoyo y acompañamiento a todos los miembros de la comunidad ya que por motivo de la pandemia todos se encuentran muy "estresados" y con necesidad especial de apoyo emocional.

A partir de las dificultades y de las medidas adoptadas, tanto docentes como gestores educativos pudieron identificar algunas oportunidades presentadas por esta situación. se identificaron subcategorías referentes a la oportunidad de capacitarse, de invertir en infraestructura y de replantear todo el sistema para realizar la migración digital. Con respecto a la capacitación, los participantes notaron que esta situación "presenta increíbles oportunidades para aplicar instrucción diferenciada... fomentar la 
creatividad por parte del alumno y del maestro". "Está brindando la oportunidad para crecer en el uso de la tecnología y poder acceder a un mundo de posibilidades”. Por otro lado, también mencionaron la oportunidad de invertir en infraestructura, especialmente tecnológica. Finalmente, varios se refirieron a la "oportunidad de reestructurar los sistemas que tenemos" de manera a cambiar desde el docente y usar la tecnología para "POTENCIAR el proceso de aprendizaje". De este modo se podrá tener "la creación de CONCIENCIA COLECTIVA" que cambie la visión sobre educación.

\section{DISCUSIÓN Y CONCLUSIONES}

Uno de los principales problemas de la pandemia del COVID-19 reside en el impacto al derecho a la educación, perjudicada fuertemente por las normas de confinamiento actuales (Cáceres-Muñoz et al., 2020). Se han cuestionado los cierres prolongados de centros educativos (Wang et al., 2020) ya que no se evidencia su contribución exacta en la transmisión del virus (Viner et al., 2020). Además, los efectos negativos se extienden desde la falta de infraestructura (Reimers \& Schleider, 2020), brechas sociales y económicas separando a aquellos con acceso a la tecnología y aquellos sin acceso (Álvarez et. al, 2020; Armitage \& Nellums, 2020; Esposito \& Principi, 2020; Li \& Lalani, 2020; Lloyd, 2020; VivancoSaraguro, 2020) y falta de habilidades para el uso eficiente de las TIC (Pérez \& Tufiño, 2020; Saheli \& Saheli, 2012). Las desigualdades exacerbadas por el COVID-19 comprenden brechas de acceso, de uso y de competencia de las TIC afectando en este caso a toda la comunidad educativa (García-Peñalvo, 2020). Tales brechas fueron evidenciadas en los resultados de este estudio ya que los docentes del interior del país fueron los que expresaron tener más dificultades en cuanto al acceso a las computadoras y a la conectividad.

En cuanto a la situación particular de los docentes, Becta (2004) encontró que existen barreras en cuanto a la utilización de las TIC en el aula, como por ejemplo falta de entrenamiento tecnológico y pedagógico, falta de acceso a herramientas y falta de tiempo. Actualmente, debido al cambio abrupto de un sistema presencial a uno virtual, la tarea de transformar contenido y adaptarlo a las clases online puede parecer abrumadora, especialmente para los docentes con menos experiencias en el manejo de las TIC (Dunlap et al., 2016). En este sentido, los gestores de las instituciones educativas indicaron que sus docentes carecen de una capacitación adecuada en el uso de las TIC para llevar adelante el proceso de enseñanza-aprendizaje de manera eficaz. El incremento de trabajo se vio reflejado en los resultados del presente estudio concordando con otro estudio que estima que planificar clases online conlleva un $14 \%$ más de tiempo que una clase tradicional y el tiempo de dedicación a la enseñanza aumenta considerablemente en tiempos de evaluación (Tomei, 2006). A esta dificultad se suma trabajar desde casa, al mismo tiempo atender a las tareas del hogar y la falta de acceso a las herramientas necesarias para la 
realización de clases (Monasterio \& Briceño, 2020; Zhang, Wang, Yang \& Wang, 2020). Todo esto se pudo evidenciar especialmente en el análisis cualitativo donde tanto docentes como gestores indicaron tener dificultades similares a las reportadas en estudios previos.

La situación actual conlleva a repensar el propósito de las clases virtuales remotas enfatizando el objetivo de proveer acceso temporal a la educación y no así imitar sistemas robustos de educación (Álvarez H. et al., 2020; Arias et al., 2020). Es decir, el enfoque educativo debe basarse en el apoyo constante a docentes (Lloyd, 2020) y asegurar la educación de los estudiantes (Daniel, 2020). En otras palabras, Soto-Córdova (2020) hace énfasis en la relación educador-educando y la importancia de las relaciones psicoafectivas para la continuidad del proceso educativo y la superación de dificultades relacionadas a la escuela. En este contexto, se pudo evidenciar que el rol del gestor educativo responde a nuevos desafíos para hacer frente a las necesidades del entorno académico ya que los gestores además de dar apoyo a la comunidad educativa y acompañar el proceso de aprendizaje tuvieron que establecer medidas financieras para salvaguardar la continuidad de la educación. Así, tanto docentes como gestores se mostraron preocupados con la calidad del proceso educativo considerando las dificultades presentadas por la situación.

Igualmente, el proceso educativo se ve afectado por la disconformidad del docente en cuanto al cambio abrupto de clases tradicionales a clases virtuales. Así mismo, otro estudio indica que tres de cada cuatro docentes no se sienten preparado para implementar nuevas TIC en el aula evidenciando la falta de capacitación y acceso a herramientas tecnológicas (Arias et al., 2020). Esto se pudo evidenciar tanto en el análisis cuantitativo como en el cualitativo en este estudio. Similarmente, en un estudio sobre retos educativos para profesores universitarios se demostró que, si bien los docentes se encontraban de acuerdo con el apoyo institucional recibido, demostraron dificultades tanto tecnológicas, pedagógicas y también socioafectivas (Sánchez Mendiola et al., 2020). De esta manera, los esfuerzos del docente deben ser acompañados con planes de "fortalecimiento para las competencias relacionadas con la seguridad informática y la comunicación y elaboración de contenido digital" (Martínez-Garcés \& GarcésFuenmayor, 2020, p. 11).

Cabe destacar que, al principio de la cuarentena, las instrucciones dadas por los entes reguladores de la educación en Paraguay (MEC, CONES) fueron percibidas como superficiales para establecer procesos pedagógicos en respuesta al cierre de escuelas. Tal situación, llevó a la incertidumbre sobre cómo enfrentar la migración digital y sobre todo como apoyar a docentes y estudiantes en este proceso. Con relación a este problema, Soto-Córdova (2020) hizo notar las dificultades que sobrellevan profesores, estudiantes y entes educativos al tratar de hacer frente a las clases virtuales, siendo la falta de lineamientos 
pedagógicos y programáticos por parte del MEC y los cambios continuos en cuanto a la implementación de nuevas estrategias, los desafíos más importantes.

La necesidad de un plan o línea de acción ante situaciones de adversidad, como la actual, es sin duda indispensable dentro de las instituciones de tal manera que aseguren no solo la continuidad del proceso educativo sino también el liderazgo por parte de los gestores educativos (Hernández, 2020). En ese sentido, el presente estudio evidencia la falta de preparación institucional considerando la escasa infraestructura tecnológica disponible y un número significativo de docentes que no se encontraban capacitados en el uso de herramientas tecnológicas antes del inicio de la cuarentena, algunos de los cuales siguieron sin recibir capacitación luego de varias semanas. Esto puede deberse a que como hacen notar Arias et. al. (2020), en el sistema de educación superior de América Latina y el Caribe, el 38\% docentes manifiesta que sus instituciones no cuentan con incentivos que promuevan el uso de tecnologías digitales. También resaltan la falta de visión por parte de las instituciones superiores a la hora de utilizar la tecnología para la mejora de la enseñanza y el aprendizaje (Arias et. al., 2020).

En un estudio sobre instituciones superiores en los Estados Unidos, el 89\% de los gestores educativos reporta que sus instituciones adoptaron medidas tecnológicas para dar continuidad a la educación. De la misma manera, entre las estrategias más útiles necesarias para la institución se mencionaron: información sobre cómo mejor apoyar a los estudiantes, mejores prácticas para apoyar a docentes que se encuentren trabajando desde el hogar y mayor acceso a materiales digitales (Johnson, 2020). Similarmente, en este estudio se pudo evidenciar la falta de materiales e infraestructura tecnológica para la realización de las clases virtuales, siendo este último necesario no solo para docentes, sino también para estudiantes.

Dentro de este contexto, el estudio refleja las estrategias de cambio de las instituciones educativas en respuesta a la pandemia tales como reducción de personal, de salario y la implementación de descuentos para los padres y estudiantes con el fin de sobrellevar la situación económica. Considerando el caso de Paraguay, las decisiones tomadas por parte de los gestores educativos al hacer frente a la crisis actual son en beneficio de la continuidad de la educación. Sin embargo, tales decisiones y medidas deben abarcar también el bienestar de los profesores y estudiantes. Si bien es cierto que son tiempos sin precedentes, debe primar la consideración de los diferentes contextos de donde provienen los profesores y estudiantes para la adecuación pertinente de los procesos educativos.

Para concluir, los resultados de este estudio muestran que a inicios de las medidas adoptadas por el gobierno las instituciones educativas tuvieron muchos inconvenientes en el desarrollo de las clases a distancia. Las principales dificultades reportadas por docentes y gestores educativos incluyeron aspectos referentes a la migración digital, tales como el limitado acceso a internet y a las TIC, así como la escasa 
capacitación en su uso por parte de todos los miembros de la comunidad, la incertidumbre ante la situación y la mantener la calidad educativa. De modo a dar continuidad a los procesos, las medidas adoptadas por las instituciones incluyeron adaptarse a las circunstancias en la medida de lo posible y tomar medidas financieras entre otras. A pesar de las dificultades, tanto gestores como docentes indicaron que esta situación al traer a la luz las diferencias existentes en la sociedad es una excelente oportunidad de replantear el sistema educativo.

Por todo esto, se puede decir que minimizar la deserción estudiantil y conservar la calidad de la educación representan los mayores desafíos para la continuidad del proceso educativo. Estos aspectos demuestran la necesidad de un mayor y mejor acceso a las tecnologías educativas por parte de los principales actores educativos del país para garantizar la igualdad de oportunidades académicas (Trujillo et al., 2020) y a la vez asegurar que los estudiantes paraguayos accedan "a una educación de calidad, en igualdad de condiciones" (Juntos por la educación, 2019, n.p.). Un estudio de seguimiento para dar continuidad a este está planificado para el final del año lectivo de manera a poder tener en cuenta cómo evolucionaron las perspectivas de los actores educativos en el transcurso del año y cómo evalúan estos la experiencia de la migración digital. 


\section{REFERENCIAS BIBLIOGRÁFICAS}

Álvarez, M., Gardyn, N., Iardelevsky, A. y Rebello, G. (2020). Segregación Educativa en Tiempos de Pandemia: Balance de las Acciones Iniciales durante el Aislamiento Social por el Covid-19 en Argentina. Revista Internacional de Educación para la Justicia Social, 9(3).

Álvarez, H., et al. (2020). La educación en tiempo de coronavirus: Los sistemas educativos de América Latina y el Caribe ante COVID-19. Banco Interamericano de Desarrollo.

Arias E., Escamilla J., López A. y Peña L. (2020). COVID-19: Tecnologías Digitales y Educación Superior ¿Qué opinan los docentes?. Centro de Información para la mejora de los aprendizajes CIMA. Inter-American Development Bank IDB. Retrieved from: https://bit.ly/3kj00tb

Armitage, R., \& Nellums, L. (2020). Considering inequalities in the school closure response to COVID-19. The Lancet Global Health, 8(5), e644. doi:10.1016s2214-109x(20)30116-9

Britez, M. (2020). La educación ante el avance del COVID-19 en Paraguay. Comparativo con países de la Triple Frontera.

Cáceres-Muñoz J., Jiménez A. S., y Martín-Sánchez M., (2020). Cierre de escuelas y desigualdad socioeducativa en tiempos del Covid-19. Una investigación exploratoria en clave internacional. Revista Internacional de Educación para la Justicia Social, 9(3e), 199-221. https://doi.org/10.15366/riejs2020.9.3.011

CEPAL, N. U. (2020). América Latina y el Caribe ante la pandemia del COVID-19: efectos económicos y sociales.

Cifuentes-Faura, J. (2020). Consecuencias en los Niños del Cierre de Escuelas por $\quad$ Covid-19: $\quad$ El papel del Gobierno, Profesores y Padres. Revista Internacional de Educación para la Justicia Social, 9(3).

CONES. (2020, Julio 29). Educación Superior del Paraguay en Tiempos de COVID-19 | Consejo Nacional de Educación Superior CONES. Recuperado de https://bit.ly/388uwAu

Dunlap, J. C., Verma, G., \& Johnson, H. L. (2016). Presence+Experience: A Framework for the Purposeful Design of Presence in Online Courses. TechTrends, 60(2), 145-151. doi:10.1007/s11528-0160029-4

Esposito, S., \& Principi, N. (2020). School Closure During the Coronavirus Disease 2019 (COVID-19) Pandemic. JAMA Pediatrics. doi:10.1001/jamapediatrics.2020.1892

García-Peñalvo, F. J. (2020). El sistema universitario ante la COVID-19: Corto, medio y largo plazo. En Universidad. Disponible en: https://bit.ly/2YPUeXU

Hernández, A. (2020). COVID-19: el efecto en la gestión educativa. Revista Latinoamericana De Investigación Social, 3(1), 37-41. Recuperado a partir de http://revistasinvestigacion.lasalle.mx/index.php/relais/article/view/2640 
Johnson, N., Veletsianos, G., \& Seaman, J. (2020). U.S. faculty and administrators' experiences and approaches in the early weeks of the COVID-19 pandemic. Online Learning, 24(2), 6-21. https://doi.org/10.24059/olj.v24i2.2285

Juntos por la Educación. (2019). Financiamiento público de la educación en el Paraguay: Notas para el debate y construcción de políticas públicas. Asunción.

Li, C., \& Lalani, F. (2020). The COVID-19 pandemic has changed education forever.

This is how. Retrieved June 22, 2020, from https://bit.ly/3dCiqkk

Lloyd, M. (2020). Desigualdades educativas y la brecha digital en tiempos de COVID-19. En H. Casanova Cardiel (Coord.), Educación y pandemia: una visión académica (pp. 115-121). Ciudad de México: Universidad Nacional Autónoma de México, Instituto de Investigaciones sobre la Universidad y la Educación.

Martínez-Garcés, J., \& Garcés-Fuenmayor, J. (2020). Competencias digitales docentes y el reto de la educación virtual derivado de la covid-19. Educación y Humanismo, 22(39), 1- 16 https://doi.org/10.17081/eduhum.22.39.4114

MEC, Ministerio de Educación y Ciencias. (2020). Plan de Educación en Tiempos de Pandemia "Tu Escuela en Casa" [Ebook]. Asunción. Recuperado de https://bit.ly/2B8i0oJ

Monasterio, D. y Briceño, M. (2020). Educación mediada por las Tecnologías: Un desafío ante la coyuntura del Covid-19. Observador del Conocimiento, 5(1), 136-148.

Pérez M., y Tufiño A. (2020). Teleeducación y COVID. CienciAmérica 2020; Vol. 9 (2)ＩISSN 1390-9592 ISSN-L 1390-681X

OEI. (2020). Organización de Estados Iberoamericanos para la Educación, la Ciencia y la Cultura. La OEI elabora la primera guía de calidad iberoamericana sobre educación a distancia. Retrieved June 23, 2020, from https://bit.ly/3g51Cqi

R Core Team (2020). R: A language and environment for statistical computing. R Foundation for Statistical Computing, Vienna, Austria. URL https://www.R-project.org/.

Reimers, F. M. \& Schleicher, A. (2020). A framework to guide an education response to the COVID-19 Pandemic of 2020. OECD. Retrieved April, 2020, vol. 14, p.

Salehi, H., Salehi, I. Z. (2012). Challenges for using ICT in education: teachers' insights. International Journal of e-Education, e-Business, e-Management and e- Learning, (1), 40.

Sanz, I., Sainz González, J., y Capilla, A. (2020). Efectos de la Crisis del Coronavirus en la Educación Superior. Organización de Estados Iberoamericanos para la Educación, la Ciencia y la Cultura (OEI). Madrid, España. Retrieved from https://bit.ly/3ipISkS

Sánchez, M., Martínez Hernández, A., Torres Carrasco, R., de Agüero Servín, M., Hernández Romo, A. K., Benavides L., Mario A., Jaimes Vergara, C. A. y Rendón Cazales, V. J. (2020). Retos educativos durante la pandemia de covid-19: una encuesta a profesores de la UNAM. Revista Digital Universitaria (RDU) Ahead of print. 
Soto-Córdova, I. (2020). La relación estudiante-docente en tiempos de cuarentena: desafíos y oportunidades del aprendizaje en entornos virtuales. Revista Saberes Educativos, (5), 70-99. Consultado de https://ultimadecada.uchile.cl/index.php/RSED/article/view/57816/61407

Tomei, J. (2006). The impact of online learning on faculty load: Computing the ideal class size for online learning . Journal of Technology and Teacher Education, 14, 531-541.

Trujillo, F., Segura, A., Fernández, M., y Jiménez, M. (2020). Escenarios de evaluación en el contexto de la pandemia por la COVID-19: la opinión del profesorado [Ebook]. Santillana. Retrieved from https://bit.ly/3iweQed

UNESCO (2020a). "COVID-19 - Education is the bedrock of a just society in the post-COVID world." Retrieves from https://bit.ly/33rpD5f

UNESCO (2020b). "Adverse consequences of school closure." Retrieved from https://es.unesco.org/node/320395

Viner, R., Russell, S., Croker, H., Packer, J., Ward, J., \& Stansfield, C. et al. (2020). School Closure and Management Practices During Coronavirus Outbreaks Including COVID-19: A Rapid Narrative Systematic Review. SSRN Electronic Journal. doi:10.2139/ssrn.3556648

Vivanco-Saraguro, A. (2020). Teleducación en tiempos de COVID-19: brechas de desigualdad. CienciAmérica, [S.1.], v. 9, n. 2, p. 166-175. ISSN 1390-9592. http://dx.doi.org/10.33210/ca.v9i2.307.

Wang, G., Zhang, Y., Zhao, J., Zhang, J., \& Jiang, F. (2020). Mitigate the effects of home confinement on children during the COVID-19 outbreak. The Lancet, 395(10228), 945-947. doi: 10.1016/s0140-6736(20)30547-x

WHO. Coronavirus disease (COVID-19) advice for the public.(2020). $\quad$ Retrieved June 11, 2020, from https://bit.ly/2YDDtyv

Grupo Banco Mundial, 2020. COVID-19: Impacto en la educación, y respuestas de política pública. Resumen Ejecutivo. Recuperado de: https://bit.ly/301GYKZ 